\title{
Focalización con y sin incidencia argumental en la formación de palabras: el aporte de algunas lenguas indoeuropeas antiguas*
}

\author{
Verónica Orqueda**
}

\begin{abstract}
Resumen
En este trabajo se analizan formaciones con intensificadores (o focalizadores) en védico y griego antiguo a la luz de tres propuestas de análisis provenientes de trabajos en torno a diferentes lenguas modernas, con el fin de determinar su posible aplicabilidad y validez en otras posibles lenguas. Aunque con diferencias, las propuestas de Felíu (2003, 2005), Mutz (2003, 2011) y König (2011) en torno al valor reflexivo y de intensificación en compuestos se centran en la existencia de la estructura argumental de tales formaciones, sea esta heredada o no de la forma base. Ahora bien, a partir de los datos encontrados en estas lenguas indoeuropeas antiguas, se puede inferir que la incidencia argumental no siempre es un requisito esencial, pues son posibles también las formaciones cuyo primer elemento funciona como focalizador que, sin embargo, carecen de estructura argumental. A partir de esto, se propone incluir de manera general estos casos como un subtipo de focalización, capaz de funcionar de acuerdo con las restricciones particulares de cada lengua.
\end{abstract}

Palabras clave: formación de palabras, reflexivos, intensificadores.

\section{Focalization with and without influence on argument structure in word formation: evidence from some ancient Indo-European languages}

\begin{abstract}
This paper analyzes word-formation with intensifiers (or focalizers) in Vedic and Ancient Greek, according to three existing proposals applied to diverse modern languages. The aim is to determine its possible applicability and validity for other possible languages. Despite their differences, the proposals by Felíu (2003, 2005), Mutz (2003, 2011) and König (2011) regarding the reflexive/intensifying value in compounds are centered around the existence of the argument structure of such formations, regardless of whether this structure inherited or not from the base form. However, data from these ancient Indo-European languages shows that the argument structure is not always an essential requisite, given that there are also formations with a focalizing first element and which, nonetheless, display no argument structure. Under this scenario, we propose to include such formations as a subtype of focalization, which would work according to the inner constraints in each language.
\end{abstract}

Keywords: word-formation, reflexives, intensifiers.

Recibido: 24-08-2016

Aceptado: 03-03-2017

\footnotetext{
* La presenta investigación se enmarca en el proyecto CONICYT.FONDECYT 3150246.

** Doctora en estudios del Mundo Antiguo por la Universidad Complutense de Madrid. Profesora Asistente en el Depto. de Ciencias del Lenguaje, Pontificia Universidad Católica de Chile. vorqueda@uc.cl
} 


\section{Introducción}

Como bien se expone en una serie de trabajos impulsados desde hace ya algunas décadas (Faltz, 1977; König y Siemund, 2000; Heine, 2005), existe un importante vínculo entre los marcadores reflexivos de una cláusula verbal y otras funciones esencialmente semánticas con correlato en la sintaxis, como, por ejemplo, la reciprocidad o la intensificación ${ }^{1}$. Este fuerte vínculo no sólo suele encontrarse en una perspectiva sincrónica, sino también diacrónica, en tanto los diferentes valores con frecuencia se encuentran en un continuo de cambio diacrónico, lo cual explica que a partir de una de estas funciones se desarrolle alguna de las otras en una determinada lengua. Así, por ejemplo, ocurriría en inglés, cuyas antiguas formas en -self (myself, yourself, etc.) habrían pasado de desempeñar un valor únicamente de intensificación hacia la incorporación de valores reflexivos, lo cual permitiría hablar hoy de formas multifuncionales a nivel sincrónico:

(1a) The president admires himself'el presidente se admira a sí mismo' (reflexivo).

(1b) The president himself wrote the speech 'el presidente mismo escribió él discurso' (no reflexivo: intensificador).

Aquí, la multifuncionalidad en inglés moderno se da como resultado de un proceso de gramaticalización a partir de un elemento que en inglés antiguo y medieval se limitaba a realzar uno de los constituyentes, adjuntándose a un pronombre en posición de objeto (König y Siemund, 2000). Con el tiempo, el realce daría lugar naturalmente a un uso reflexivo, es decir esencialmente gramatical, en tanto es frecuente que tengan lugar situaciones con una serie de rasgos compartidos, como, por ejemplo, un alto grado de animacidad del primer argumento y una gran incompatibilidad con verbos de estado, al menos en varias lenguas modernas²:

Given that actor-oriented intensifiers oppose propositions to alternative propositions in which the actor-role is assigned to some individual other than the referent of the associated NP,

\footnotetext{
1 Si bien a lo largo de este trabajo los términos "intensificador" y "focalizador" pueden entenderse como equivalentes, la bibliografía consultada tiende a preferir el primero, mientras que, por mi parte, considero que el segundo puede ser menos ambiguo, ya que refleja la oposición entre posibles alternativas referenciales evitando la posible confusión con los también llamados "cuantificadores". 2 Nótese que esto se aplica a los intensificadores de tipo "orientados al actor" (o intensificadores adverbiales) y no a los adnominales, que sí permiten la intensificación de elementos inanimados, como por ejemplo “la puerta misma de la casa estaba rota” (véase Gast y Siemund, 2006).
} 
they can be said to emphasize the agent status of a referent in a given predication. (Gast y Siemund, 2006, pp. 359).

A partir de fenómenos de este tipo, se ha intentado llevar la investigación al proceso de formación de palabras con el objetivo de identificar si también aquí es posible el desarrollo de marcadores reflexivos a partir de otras funciones relacionadas, y si pueden darse casos de multifuncionalidad. Como aliciente de tal hipótesis no sólo se encuentra la estrecha relación en el funcionamiento de cláusulas verbales y ciertas formaciones de palabra, especialmente las nominalizaciones, sino también la multifuncionalidad de algunas formaciones en un nivel sincrónico. Dado el estrecho vínculo entre este tipo de formaciones de palabra (es decir, formaciones como el inglés self-esteem 'autoestima') y las cláusulas verbales, es esperable la posibilidad de que las primeras contengan una determinada estructura argumental ${ }^{3}$, aunque, como veremos, ésta podría no ser obligatoria para todos estos tipos de formaciones.

Es importante señalar que el análisis de estructuras argumentales en el ámbito de la formación de palabras ha recibido atención dispar, tanto en lo referente a posibles enfoques teóricos —es mucho más amplia la tradición generativista (Chomsky, 1970; Grimshaw, 1990; Alexiadou, 2001, entre otros), que la funcional ${ }^{4}$ o cognitivista-como en cuanto al tipo de formaciones, pues la mayoría de las investigaciones se centran más en la estructura de sustantivos y sus distinciones (por ejemplo, eventuales vs. resultativos), y especialmente de nominalizaciones deverbativas, y menos en otras categorías como la adjetival o la preposicional. En particular, hay que decir que en el ámbito del español los trabajos son más descriptivos y exploratorios que investigaciones encauzadas únicamente dentro de un marco teórico, como es el caso de Picallo (1999) y Felíu (2003).

El hecho de que no sólo las cláusulas verbales sino también las formaciones de palabras mediante prefijación (o composición, en ciertas lenguas) pueden ser reflexivas o presentar una función cercana es evidente en casos como el español:

\footnotetext{
3 En este trabajo se hará referencia al concepto de estructura argumental como la codificación de información léxica de tipo y número de argumentos que un elemento acepta y de la información necesaria para comprender su organización sintáctica. Como afirma Levin (2013, s/n, párr. 1), "the term 'argument structure' is used to refer to the lexical representation of argument-taking lexical items-typically verbs, but also nouns (especially nominalizations), adjectives, and even prepositions-that specifies sufficient information about these items' arguments to allow their syntactic realization to be determined".

4 Para un análisis funcional de las estructuras argumentales del sintagma nominal en español véase especialmente el trabajo de González (2002).
} 
(2a) [...] la meta común que todas promocionan es la autoayuda (CREA).

(2b) [...] también contempla la organización y autogestión de la comunidad... (CREA).

Si bien tanto autoayuda como autogestión pueden considerarse biargumentales, pues llevan asociados al menos dos participantes semánticos, en los contextos propuestos sólo el primero puede interpretarse como reflexivo (equivalente a "ayudarse a uno mismo"), mientras que los referentes de los argumentos seleccionados por autogestión están diferenciados, dado que el grupo preposicional se presenta, a diferencia de los esperado, como el argumento interno pero no externo.

Este fenómeno no es único del español (lengua en la que se centran los fundamentales trabajos de Felíu (2003, 2005)), sino que se encuentra también en otras lenguas, como, por ejemplo, en italiano (Mutz, 2003, 2011) y en muchas otras lenguas del mundo, como queda claro en el acercamiento más bien tipológico de König (2011), dedicado al análisis de posibles restricciones y características interlingüísticas; de allí que podamos esperar que el mismo fenómeno pueda encontrarse en otras lenguas, como las que aquí analizaremos.

Estos trabajos presentan tanto diferencias como similitudes. Entre las últimas, cabe destacar que no se restringen al fenómeno de la nominalización deverbativa para explicar estas formaciones, que también pueden ser verbales o adjetivales, como en autogenerado, autodestructor, autorremover. Nótese que si bien es frecuente que las nominalizaciones conserven la estructura argumental propia de la base de la que derivan (Grimshaw, 1990), tal como sucede en español con percibir (X, Y) > autopercepción $(\mathrm{X}, \mathrm{X})$, en este tipo de formaciones no se trata de un requisito excluyente, como lo demuestran formaciones del tipo autogol (X, $\mathrm{X})$, que también es biargumental, a pesar de que su base, gol, no lo es. Este panorama más amplio con respecto l de las nominalizaciones hace que los trabajos de estos tres autores sean fundamentales para intentar entender el funcionamiento equivalente en otras lenguas, tal como veremos aquí, ya que evidentemente el fenómeno tampoco se restringe a nominalizaciones. Ahora bien, en cuanto a las diferencias, éstas permiten reflexionar acerca de cómo llevar estos acercamientos a otras lenguas. Por esto, en las próximas páginas veremos las características principales de tales acercamientos para después considerar su posible aplicación al corpus seleccionado en védico y griego antiguo. 


\section{Propuestas previas}

El primer acercamiento a comentar es el de König (2011), que esencialmente lleva al plano de la formación nominal propuestas previas en torno al funcionamiento de reflexivos e intensificadores en cláusulas verbales, como en König y Siemund (2000). Brevemente, en estos trabajos los intensificadores pueden clasificarse en las siguientes categorías (extraídas y ejemplificadas a partir de König, 2011):

(a) Adnominales: The Pope himself will come to the rally (in contrast to one of his clergy) 'El Papa mismo vendrá a la Carrera (a diferencia de uno de sus clérigos)'

(b) Adverbales ${ }^{5}$ exclusivos: Mrs. Dalloway wanted to buy the flowers herself (alone, no delegation, no help) 'La Sra. Dalloway quería comprar las flores por sí misma (sola, sin ayuda)'.

(c) Adverbales inclusivas: I cannot give you any money. I am a little short of cash myself(also) 'No puedo darte nada de plata. Yo también estoy corta/o de efectivo'.

(d) Atributivas: John's own death surprised me more than that of his brother (in contrast to someone else's death) 'La muerte del propio John me sorprendió más que la de su hermano (en contraste con la muerte de otro)'.

(e) Reflexivas: John assesses himself 'Juan se evalúa a sí mismo'.

Desde esta línea, y ya en el plano de la formación de palabras, König (2011) los denomina de manera genérica "compuestos" (reflexive compounds) con el fin de no diferenciar entre los dos tipos de formaciones en que se clasificarían las lenguas del mundo: por un lado, aquellas en las que el desarrollo se produce por derivación, como el español, en tanto el primer elemento es un prefijo y no un lexema que pueda formar palabras de manera independiente ${ }^{6} ; \mathrm{y}$, por otro, aquellas en las que se desarrolla

5 Aquí sigo la sugerencia de un evaluador anónimo que sugiere evitar la traducción 'adverbial' por la eventual confusión con la categoría de palabras. Sin embargo, debe quedar claro que en los trabajos que aquí seguimos utilizan el mismo término en la versión original en inglés (adverbial), sin temor a una posible confusión; de allí que originalmente prefiriéramos el equivalente en español.

6 Nótese que, en español, mientras que los valores reflexivos y de focalización se encuentran asociados al prefijo (auto-- ), existe un lexema homónimo (auto- ${ }_{2}$ ) que procede de la evolución fonética de acto, así como también un lexema creado por acortamiento a partir de automóvil que permite la formación de compuestos del tipo autovía o autoescuela, así como también un tema culto, base de confijaciones como autismo o autopsia (véase especialmente Felíu, 2003, 49-52). Dado que formas como estas últimas son préstamos completos y no dan cuenta de la productividad del sufijo, ya que no pueden descomponerse de esa manera en español, tales ejemplos se han dejado de lado para este análisis, en el que nos limitamos a los casos de auto- $_{3}$, es decir a aquellos en los que el prefijo se adjunta de manera productiva a lexemas preexistentes en la lengua en cuestión, más allá de su origen culto. 
por composición, en tanto el segmento analizado sí funciona como palabra independiente, como ocurre con selbst en alemán, que de manera libre puede ser un adverbio de focalización. Al llevar la clasificación de intensificadores en cláusulas verbales a la formación de palabras, la lista de posibilidades se reduce esencialmente a los siguientes:

a) Adnominales construidos en conjunto con el marcador reflexivo, en cuyo caso hay coindexación de los referentes de los constituyentes, como en la autocrítica de Juan, donde se enfatiza que el referente del elemento más pacientivo es, contra lo esperado, el mismo del elemento más agentivo. Ejemplos de otras lenguas también pueden ser el alemán Selbsthass 'autoodio', el francés autosatisfaction 'autosatisfacción' y el inglés self-addressed 'autodirigido' (como en a self-addressed envelope 'un sobre dirigido a uno mismo') (ejemplos de König, 2011).

b) Adverbales exclusivos, que tienen lugar cuando el referente focalizado y contrapuesto corresponde no sólo al paciente, como se esperaría, sino también al agente. Esto ocurre en casos como en alemán Selbstaufgabe 'autoabandono' o en inglés self-induced, self-administration (como en this village has self-administration 'este pueblo tiene autoadministración') (ejemplos extraídos de König, 2011). Como el propio autor señala, aquí podría percibirse un énfasis en la acción descrita, a diferencia del grupo anterior.

La prueba crucial para distinguir entre uno y otro consistiría en considerar si el referente que ofrece el contraste con otros potenciales es el del agente (entonces, adverbal) o del paciente (entonces, adnominal) de la estructura argumental en la base de la derivación, ya que el autor considera que una propiedad compartida por muchas lenguas (al menos las que él analiza ${ }^{7}$ ) es que el segundo segmento del compuesto endocéntrico es generalmente una nominalización o adjetivación deverbativa, con raras excepciones, como el alemán Eigenkapital 'equidad', (Therapie), Eigenblut 'autohemoterapia, terapia de la propia sangre', Eigenname 'nombre propio', en las que el uso de eigen en lugar de selbst estaría conectado a la posibilidad de anexarse a sustantivos que no son nominalizaciones. Fundamentalmente, este tipo de formaciones, mucho menos frecuentes según König (2011), corresponderían a casos atributivos, con una laxa relación de posesión. Lamentablemente, este acercamiento no va mucho más allá en el análisis de ejemplos como estos, tan especial

7 Inglés, finés, francés, alemán, griego, húngaro, italiano, mandarín y ruso. 
por no presentar con claridad redes argumentales con agente y paciente.

Por su parte, en los trabajos de Felíu (2003, 2005), en torno al español, encontramos algunos puntos en común con el acercamiento anterior, como por ejemplo una aproximación más bien bipartita de las formaciones:

Junto a este uso reflexivo de auto-, que consideramos primario ya que se documenta en la mayor parte de las piezas léxicas formadas con este prefijo, existe también un uso secundario no reflexivo, que denominaremos 'modal enfático.' En las relaciones en las que el prefijo auto- presenta este segundo valor no existe una relación de reflexividad, sino que se resalta la ausencia de una causa externa al evento. (2003, p. 54).

Entre los ejemplos que la autora utiliza para esta clasificación, se pueden citar las formaciones reflexivas como autoevaluativo, autoconceder o autopublicidad y las formaciones modales enfáticas como autoaplicador, autodegradar o autooxidable. Según esta propuesta, en una misma categoría (la de modales enfáticas) quedarían agrupados los casos con y sin una estructura argumental no reflexiva. Y en cambio, la subclasificación de las modales enfáticas respondería a la clasificación de las bases, según rasgos semánticos como el grado de animacidad del sujeto; de esta manera, se distinguirían formaciones como autogestionar, autoliquidar, que podrían transformarse en reflexivas gracias al rasgo [+animado] del sujeto, de otras como autoalimentarse, autodisparador, en las que el prefijo pondría de manifiesto la espontaneidad del evento.

Sin duda, una de las observaciones más acertadas de este segundo acercamiento es la insistencia en que no es necesario recurrir a una estructura argumental preexistente a la base, puesto que en el prefijo sería suficiente para establecer los requisitos argumentales, como queda claro con el ya mencionado caso de autogol. Sin embargo, fuera de alcance de este acercamiento quedan los posibles casos atributivos y sin incidencia argumental, debido a que parecería que tales casos no existen en español ${ }^{8}$.

Por último, Mutz (2003, 2011), cuya investigación se centra especialmente al italiano, pero de manera extensible también a otras lenguas

8 En efecto, como parte de esta investigación se realizó un rastreo histórico de estas formaciones y los pocos casos atributivos corresponden al lenguaje técnico. 
románicas, clasifica las formaciones en tres categorías, según la función del prefijo en cada caso:

a) auto - reflexivo: autodistribuzione 'autodistribución',

b) auto - anticausativo: autoaccensione 'autoencendido', autoscioglimento 'autodisolución', que carecen de la intervención de un agente,

c) auto - focalizador: autoconsumo, autogestione 'autogestión', cuya función es la de focalizar el referente que realiza la acción indicada en la base derivacional y que suele referir fundamentalmente a nombres de acción y de instrumento.

Esta distinción muestra que en los casos analizados por esta autora el prefijo también incide en la estructura argumental de la forma resultante. Lo interesante aquí es que el focalizador y el anticausativo se diferenciarían desde el comienzo y sólo el segundo sería capaz de alterar la estructura argumental de la formación resultante a partir de aquella de la base. Con respecto a auto $_{3}$, la autora (Mutz, 2003, 2011) sostiene que solamente puede focalizar el agente y no otros actantes, como el tema o la meta, por ejemplo.

Si bien, como admiten todos estos autores, es importante no perder de vista que el significado de estas formaciones es siempre muy complejo y que la posibilidad de ambigüedad e interferencia es alta, parece plausible intentar llevar tales clasificaciones a otras lenguas e intentar ver qué desafíos presentan en cada caso. En la siguiente sección se presentará el caso de dos lenguas antiguas para poder evaluar la pertinencia de estas propuestas. Como se verá, tener en cuenta el contexto de aparición resulta siempre útil, ya que muchas veces una misma formación puede tener más de una interpretación, y con frecuencia es el contexto de uso el que permite preferir un valor sobre otro.

\section{Védico y griego antiguo}

En primer lugar, es necesario mencionar que las aquí analizadas ${ }^{9}$ son sólo dos de las lenguas indoeuropeas, antiguas y modernas, estudiadas como

9 Una versión previa de este manuscrito incluía también el análisis del latín, a partir de datos extraídos del TLL. Sin embargo, dado que los ejemplos encontrados correspondían todos a préstamos o calcos del griego y no se puede considerar un fenómeno productivo de formación de palabras, he decidido eliminar esa sección. En todos los casos, no sólo se ha tomado en cuenta la traducción a partir de los diccionarios, sino que también se ha cotejado su uso de primera fuente, es decir en su contexto de uso. 
parte de una investigación más amplia centrada precisamente en torno a estas formaciones, por lo que su selección para este trabajo únicamente se justifica como medios de ejemplificación de la presente propuesta, sin que esto implique la necesidad de ofrecer mayores generalizaciones por el momento respecto del funcionamiento de este fenómeno en las lenguas indoeuropeas en general, ni las antiguas en particular. Ahora bien, es importante mencionar que, como otras lenguas antiguas de esta misma familia, éstas no sólo se caracterizan por sus complejos sistemas de flexión, sino también por ser lenguas en las que la composición es un fenómeno altamente productivo para la formación de palabras. Como se verá, en ambas lenguas el fenómeno se da por composición y no por derivación.

\section{a. Védico ${ }^{10}$}

La formación de palabras en cuestión se realiza con svá-, y en ciertos casos también con svayám, elementos cuya etimología es diferente de la del griego autós. Al igual que en griego, en védico svá puede funcionar como palabra independiente, con el valor de un posesivo, posiblemente enfático (véase Orqueda (2017), en prensa):

(3) subhvàh svấ abhișțayah 'Sus propios poderes superiores'.

En la formación de palabras con $s v a{ }^{-11}$ encontramos casos como svakșatra 'que gobierna o guía por/a sí mismo' (a partir de kṣatra 'poder, guerrero' y éste de kși 'tener poder, dominar'), svarāj 'que gobierna por sí mismo' (a partir de rāj 'gobernante' y éste de rāj 'gobernar'), svajā 'autocreado, autoproducido' (a partir de jā 'nacido, creado' y éste de jan 'procrear, nacer'), svagopa 'guardián de sí mismo' (a partir de gopa 'guardián' y éste de gup- 'proteger'), svayāvan 'que se mueve por sí mismo, que va por su propio camino' (a partir de yāvan 'que va' y éste de yam 'ir'), svagūrta 'propio regocijo' (a partir de gūrta' 'regocijo'). Como se puede ver, son deverbativas en casi todos los casos, lo cual puede permitirles la conservación de una determinada estructura argumental, a pesar de que también puedan cambiarla. Es decir, las formaciones pueden no coincidir

10 El corpus analizado está compuesto por el Rgveda (RV) y el Atharvaveda (AV). Téngase en cuenta que la selección del corpus busca obtener solamente una muestra representativa. Dentro de esta muestra, las diferencias entre RV y AV no fueron significativas, por lo que la elección del RV para ejemplificación se debe únicamente a razones prácticas.

11 Los ejemplos de formaciones con svayám se han omitido aquí pues no presentan diferencias significativas respecto de svá- en cuanto a los criterios de selección de base.

12 Cf. gratus, en latín. 
en cuanto al número y tipo de argumentos con las bases sin composición, pero en ambos casos existe una estructura argumental definida.

En cuanto a las posibles funciones encontramos primero casos en los que svá da lugar a una formación enfática atributiva como svadhā 'el propio poder, el propio lugar', cuya base, $d h \bar{a}$ 'ubicación, riqueza', si bien posee estructura argumental (claramente a partir de su origen verbal), no implica necesariamente una relación posesiva:

(4) yádi me rāránaḥ sutá ukthé vā dádhase cánah ārấd úpa svadhāa gahi 'Si te regocijas con mi exprimido (soma) u obtienes placer en mi discurso, acércate desde lejos mediante tu propio poder' (RV. 8.32.6).

En (4), la relación atributiva se establece entre $d h \bar{a}$ y aquel a quien se dirige el himno, focalizando pragmáticamente tal referente en detrimento de otros posibles.

Entre otros casos argumentales encontramos también una posible diferenciación entre monoargumentales y biargumentales, que dan lugar a interpretaciones de focalización del agente y a reflexivos respectivamente, con una leve mayoría de los casos no reflexivos. Ejemplos:

(5) áto vayám antamébhir yujānáh svákṣatrebhis tanvàh śúmbhamānāh 'Por esto, unciendo nosotros (los carros) a los (caballos) más cercanos que se conducen solos y embelleciendo nuestros cuerpos' (RV 1.165.5ab).

(6) ánu svajā́m mahiṣáś cakṣata vrấm ménām 'el poderoso miró fijamente a la aurora, muchacha autocreada' (RV 1.121.2c).

Si bien svakșatra en (5) también podría dar lugar a una interpretación reflexiva (caballos que se guían a sí mismos), en este caso parecen focalizar sólo la agentividad del actor, gracias a la referencia a los carros. De esta manera, son los caballos mismos los que guían los carros. Por su parte, lo relevante en (6) es que la aurora se crea a sí misma, dado que la estructura argumental de este elemento requiere necesariamente dos participantes y no hay ningún otro en el contexto que pueda descartar la correferencia.

Considerando estos casos a través de las propuestas mencionadas al principio, se puede ver, por un lado, que es acertada la consideración de König (2011) de intensificadores atributivos, pues efectivamente los encontramos en esta lengua; por otro lado, la diferenciación entre casos de focalización de un agente y casos de ausencia del agente, planteada por Mutz (2003, 2011), también es pertinente para el védico. Respecto 
de eventuales casos sin incidencia argumental, al no haber encontrado ejemplos en esta lengua no es posible descartar ninguno de los análisis mencionados, más allá de que unos se ajusten mejor que otros.

\section{b. Griego $0^{13}$}

En esta lengua, las formaciones se obtienen a partir de la unión de un lexema libre con auto-, que corresponde a la forma sin flexión de autós $(\alpha v i \tau o ́ \varsigma)^{14}$ 'mismo, propio', el demostrativo intensificador prototípico en la lengua clásica (Mocciario, 2013):

(7) $\alpha \dot{\tau} \tau o ́ \varsigma ~ \varepsilon ́ \gamma \omega ́$ 'yo mismo/en persona'.

Como palabra independiente, este intensificador es fundamentalmente adnominal y a partir de tal valor se desarrolla, entre otros posibles, como parte del marcador reflexivo (Puddu, 2005). En formación de palabras, se trata de un elemento altamente productivo y que ofrece muy pocas restricciones de selección, en tanto encontramos casos como: autóboulos 'que obra por su propia voluntad', autogenès 'existente por sí mismo, nacido de sí mismo', autognómōn 'según el propio juicio', autodídaktos 'autodidacta, que se enseña/aprende de/a sí mismo', autotragikós 'que es totalmente trágico', autokrátōr 'autoridad absoluta, que tiene el control', autophónos 'que mata a su propia familia', autoetés 'ese mismo año', autourgós 'que trabaja o actúa por sí mismo', autophuếs 'natural, según la propia naturaleza', autokasignétēe 'la propia hermana', autókomos 'naturalmente, con su mismo follaje', autómatos 'que se mueve por sí mismo, por su propia voluntad, autonuxi 'la misma noche', automémōn 'que sufre las propias penas', autotrígōnon 'triángulo en sí mismo'.

Un análisis detallado de algunos de estos casos en sus contextos permite ver que existe una amplia variedad de valores en este tipo de formaciones. Así, por ejemplo, encontramos, primero, que la formación

13 Corpus analizado: Odisea, Ilíada (Homero), Teogonía, Los trabajos y los días (Hesíodo), Siete contra Tebas, Suplicantes, Agamenón, Persas (Esquilo), Categorías, De la generación y corrupción, Historia de los animales, Poética (Aristóteles), Nubes, La paz, Lisístrata, Las ranas (Aristófanes), Eutifrón, Apología de Sócrates, Menón, República, Leyes (Platón), Filípicas, Sobre la corona, Sobre la falsa embajada, Contra Midia, Contra Aristócrates (Demóstenes). Téngase en cuenta que aunque la selección de ejemplos pueda parecer poco representativa, el análisis hecho demuestra que ni la variación diacrónica ni textual ofrecen diferencias significativas, por lo que se ha optado simplemente por presentar aquellos ejemplos que puedan resultar más claros. En cambio, sí se encuentran diferencias entre estas variedades del griego y el posclásico, razón por la cual este último ha sido excluido en el presente trabajo y se incluye en un trabajo en preparación dedicado exclusivamente al conjunto de las lenguas antiguas.

14 En esta sección se mantendrá el alfabeto griego para los ejemplos correspondientes, pero se preferirá la transliteración en el caso en que las palabras citadas formen parte del cuerpo del texto. 
de reflexivos es poco productiva, ya que pocos casos lo son de manera inequívoca, como en (8):

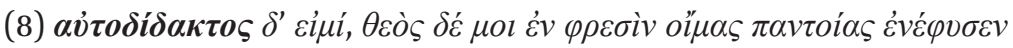
'Soy autodidacta, y un dios me implantó toda clase de cantos en la mente' (Hom. Od. XXII, 347).

Aquí, autodidaktos es bivalente, en tanto la misma estructura parece exigir como parte de la red un participante que instruye y otro que es instruido. En este caso en particular, la formación reproduce la estructura argumental heredada de su base didaktós 'que puede ser enseñado o aprendido', permitiendo en ambos casos la existencia de dos participantes.

En segundo lugar, encontramos unos pocos casos como en (9):

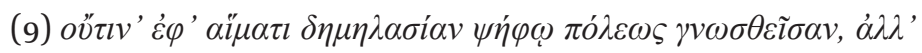

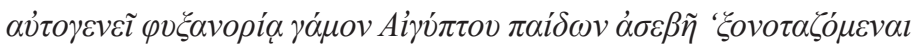
'sin que el voto del pueblo nos hubiera condenado al destierro por un delito sangriento, sino que por una aversión nacida por sí misma (autoexistente), abominando la impía boda con los hijos de Egipto'. (Aesch., Suppl., 8).

Como se ve, la aversión existe o nace por sí misma, sin una fuerza agentiva determinada. Dentro del marco de la primera propuesta, este tipo de construcciones debería categorizarse como adverbal. Sin embargo, esta misma categoría es confusa, ya que la estructura argumental de esta formación sugiere la existencia de un único participante más bien pacientivo que agentivo, en tanto evoca un evento inacusativo; es decir, aquello que existe por sí mismo y no lo hace activa y agentivamente, como parecería ser la propuesta para los adverbales exclusivos. Por su parte, tampoco resulta aplicable la propuesta de Felíu (2003) para el español, ya que dentro de este marco, en tanto modal enfático, no habría diferencias con respecto a los casos en que la estructura argumental sugerida cuenta con un participante agentivo. En este sentido, sólo la propuesta de Mutz (2003, 2011), que considera de manera diferenciada las formaciones anticausativas, parecería explicar mejor este tipo de formaciones en griego.

En tercer lugar, encontramos formaciones como autourgós y autóboulos, en (10):

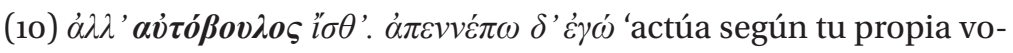
luntad, pero yo lo prohibo' (Aesch., Septem., 1053).

Estos casos, a diferencia de los anteriores, presentan una estructura monoargumental en la que el participante sí es agentivo. Es importan- 
te señalar que en el caso de autóboulos, esta formación funciona como adjetivo y como tal puede servir de atributo a un referente animado que realiza una acción; de allí la traducción ofrecida como 'aquel que actúa por su propia voluntad, según su propia determinación', para indicar la interpretación de este caso como uno de focalización. Sin embargo, se trata de una formación nueva, ya que el griego clásico no registra el adjetivo *boulós, pero sí el sustantivo boule’ ‘decisión, determinación'. Es importante notar que si la formación fuera autoboulé podría tratarse de un intensificador atributivo 'su propia voluntad', pero en el contexto visto la situación es algo diferente debido a la presencia de un verbo liviano.

Por último, hay un importante número de casos en los que se puede ver que ni la formación de base ni la resultante poseen una estructura argumental pero sí se mantiene el valor de intensificación agregado por auto-. Esto se ve en casos como autokasignétēe, autoetés y autotragikós:

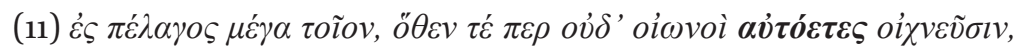

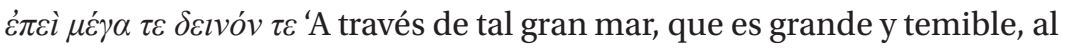
cual ni las aves cruzan en un mismo año' (Hom., Od. III, 322).

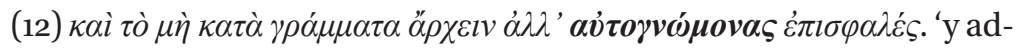
ministrar no a través de un código escrito sino de uno inestable a propia discreción' (Aristóteles, Política, 2.1272a).

En estos casos, ni las formas base ni las construcciones resultantes poseen estructura argumental, pero la adjunción de auto- indica igualmente la focalización de un referente específico en detrimento de otros. Ahora bien, dentro del conjunto de estas construcciones, en las enfáticas atributivas el referente focalizado forma parte de una estructura posesiva, de allí la posible perífrasis 'el/la propio/a x', mientras que en los restantes, como en (11), el efecto producido es simplemente la focalización del sustantivo en cuestión. También, en este caso se trata de una formación nueva, ya que la lengua no registra la forma etés, sino étos 'año', que también carece de red argumental.

En este sentido, se puede hablar de fenómenos de focalización sin incidencia argumental, de manera parecida a procedimientos que en otras lenguas indoeuropeas antiguas se dan por sufijación, especialmente con - pte en latín y - pat en hitita, ambos con el mismo origen etimológico. Este tipo de formaciones, consideradas como raras excepciones por König (2011) no son abarcadas por Mutz (2003) y Felíu (2005), ya que no son propias de las lenguas estudiadas por ellas. Esto deja ver que si bien estas aproximaciones son suficientes para tales lenguas, desde un 
punto de vista tipológico es necesario considerar una eventual apertura del panorama.

\section{Discusión y conclusiones}

El análisis de estas dos lenguas permite ver que mientras que el rasgo de focalización o intensificación es evidente, no siempre lo es la incidencia del segmento en una posible estructura argumental. Esto lleva a pensar que al analizar otras lenguas es necesario tener en cuenta nuevos criterios, ya que aquellos con los que contamos hasta ahora no son del todo suficientes. A partir de lo visto con las diferentes propuestas y los casos encontrados, podemos pensar que a la tripartición de formaciones (a) reflexivas (ingl. self-regulation), (b) anticausativas (esp. autoaplicador) y (c) de focalización, es necesario agregar una subdivisión inicial de estas últimas según sean con o sin estructura argumental. Mientras que al primer subtipo corresponderían los casos de focalización del agente (esp. autogestión de los negocios, véd. svakșatra), el segundo admitiría tanto los casos atributivos (alem. Eigenblut, gr. autokasignétē) como los de intensificación no atributiva, vistos en griego (autoetés). A su vez, estos dos últimos se diferenciarían no sólo en la posibilidad o restricción de una relación posesiva, sino también en que los atributivos pueden ser tanto deverbativos como no deverbativos, mientras que los de intensificación no atributiva serían únicamente formaciones no deverbativas. En este sentido, este último subgrupo es el único que ofrece restricciones en cuanto al origen de las formaciones, lo cual ocurre en algunas lenguas, pero no en otras. Así, por ejemplo, queda claro que el español no admite formaciones ni siquiera a partir de sustantivos concretos $\left({ }^{*}\right.$ automes $a^{15}$, por ejemplo), pero el griego sí, a pesar de que en el ámbito de los adjetivos el español técnico sí se admitirían algunas formaciones como:

(13) $[. .$.$] con este vendaje hasta realizar un injerto autodérmico (CREA).$

(14) [...] establecieron que el entrenamiento autogénico era igualmente efectivo que la relajación (CREA).

A modo de conclusión se puede decir que si los acercamientos vistos ofrecen importantes herramientas de análisis para este tipo de formaciones, en buena medida se centran en casos en los que existe una estructura 
argumental, dejando de lado los casos en los que no. Así, estas propuestas explican bien las formaciones con intensificadores en las que se focaliza el actor de una acción (focalizadores), aquellas en las que se resalta la ausencia de una fuerza (anticausativas) y aquellas en las que los dos argumentos principales se encuentran coindexados, pero no aquellas formaciones en las que el segmento no incide en absoluto en la estructura argumental. Para esto, sería interesante proponer una categoría que permitiera incluir tales casos. Como se ha intentado mostrar, una posibilidad para conseguirlo sería considerar un subtipo de focalizador sin incidencia en la estructura argumental.

Por supuesto, la inclusión de este tipo de casos no puede determinar la elección definitiva de un marco teórico y metodológico en detrimento de otros para analizar datos provenientes de otras lenguas, sean o no de la misma familia lingüística (nótese que los que agregamos en estos trabajos forman parte del grupo lingüístico indoeuropeo, el mismo de la mayoría de los analizados por los autores citados, lo cual podría influir en la cercanía en cuanto al comportamiento. Sin embargo, se espera que las observaciones hechas aquí puedan aportar a futuras investigaciones que busquen analizar este tipo de formaciones desde una perspectiva comparativa más amplia.

\section{Bibliografía}

Alexiadou, A. (2001). Functional structure in nominals. Nominalization and ergativity. Ámsterdam: John Benjamins.

Chomsky, N. (1970). Remarks on nominalization, en Jacobs, R. y Rosenbaum, P. (eds.): Readings in English transformational grammar (pp. 184-221). Boston: Ginn.

CREA = Real Academia Española: Banco de datos (CREA) [en línea]. Corpus de Referencia del Español Actual. [http://corpus.rae. es/creanet.html].

Faltz, L. (1977). Reflexivization: a study in universal syntax, Nueva York: Garland.

Felíu, E. (2003). Morfología derivativa y semántica léxica: la prefijación de auto-, co- e inter-, Madrid: Ediciones de la UAM.

. (2005) Los sustantivos formados con el prefijo auto- en español: descripción y análisis, Verba, 32, 331-350. 
Gast, V. y Peter S. (2006). Rethinking the relationship between SELFintensifiers and reflexives, Linguistics, 44(2), 343-381.

González, C. (2002). Funciones específicas en el sintagma nominal español (Tesis de magíster). Pontificia Universidad Católica de Chile. Santiago de Chile.

(2002). Funciones nominales en el sintagma nominal español. Tesis de magíster. Santiago: Pontificia Universidad Católica de Chile.

Grimshaw, J. (1990). Argument structure. Cambridge, Mass.: MIT Press.

Heine, B. (2005). “On reflexive forms in creoles”, Lingua, 115(3), 201-257.

König, E. (2011). Reflexive nominal compounds, Studies in Language, 35(1), 112-127.

König, E. y Peter, S. (2000). Intensifiers and reflexives - a typological perspective, en Frajzyngier, Z. y T. Curl (eds.): Reflexives: forms and functions, Ámsterdam: John Benjamins, 41-74.

Levin, B. (2013). Argument structure, en M. Aronoff (ed.): Oxford Bibliographies in "Linguistics". Nueva York: Oxford University Press [en línea].

Mocciaro, E. (2013). “Intensifiers, en Georgios Giannakis (ed.): Encyclopedia of Ancient Greek Language and Linguistics, Managing Editors Online Edition.

Mutz, Katrin. (2003). Le parole complesse in 'auto' nell'italiano di oggi, en Maraschio, N. y T. Poggi Salani (eds.): Italia linguistica anno Mille. Italia linguistica anno Duemila. Atti del XXXIV congresso internazionale di studi della SLI. Firenze, 19-21 novembre 2000. Roma: Bulzoni, 649-664.

. (2011). Auto- and entre-versus(?) se. Remarks on interaction and competition between word formation and syntax, en Nolda, A. y O. Teuber. (eds.): Syntax and Morphology Multidimensional, Berlín: Mouton de Gruyter, 239-258.

Orqueda, V. (2017). Svá- como intensificador en védico, Onomázein, 36, 159-182.

Picallo, C. (1999). La estructura del sintagma nominal: las nominalizaciones y otros sustantivos con complementos argumentales, en Bosque I. y V. Demonte (dirs.): Gramática descriptiva de la lengua española. Madrid: Espasa, 363-394. 
Puddu, N. (2005) Riflessivi e intensificatori: greco, latino e le altre lingue indoeuropee. Pisa: ETS.

TLL = Thesaurus Linguae Latinae. (2000-) Munich y Leipzig. Edición electrónica. 